\title{
FOUR PULSARS WITH NEW FACES
}

\author{
LESZEK A. Nowakowski \\ Department of Physics, University of Puerto Rico at Mayaguez
}

\begin{abstract}
This paper presents results on four radio pulsars observed at Arecibo. Three pulsars have been discovered as new mode switching pulsars. In two of them the switching effect is correlated with a dramatic change of the amplitude of the core component, suggesting that the change occurs not only in the location of the emission region, but also in the efficiency of the emission mechanism. In another pulsar evidence is presented for the existence of a third (possibly a core) component which is hidden between the two conal components of the average profile. Previously unknown drifting subpulses and at least two new components have been found in one of the three mode switching pulsars.
\end{abstract}

\section{Introduction}

Radio pulsars with different types of the average profiles have been studied in order to find new objects with mode-switching behavior and to investigate a possible relationship between mode switching and the location of the emitting regions in the magnetospheres of pulsars. Such a relationship has been noticed in some pulsars and indicates a possibility that mode switching arises when the emission region changes its altitude above the surface of the neutron star. Mode changing is undoubtedly related to the mechanism generating the observed radiation from the neutron stars. As a part of the emission mechanism, mode changing will indicate how the emission mechanism works and how to interpret the received pulsar radiation. In particular, the problem of if and how the core and conal radiation are related to each other is very intriguing. Solving that problem may answer the question whether we have two different but related radiation mechanisms, or if core and conal components are generated by the same mechanism and acquire different characteristics because of different geometry and magnetospheric conditions.

\section{Observations}

This paper presents analysis of observations made with the Arecibo radio telescope at $430 \mathrm{MHz}$. PSR $0540+23$, PSR $0611+22$ and PSR $1133+16$ were observed as part of a search for new pulsars with mode-switching behavior. A $16 \times 250 \mathrm{kHz}$ filter bank was used with a time constant of $1 \mathrm{~ms}$. Dispersion was removed off-line. Observations of PSR $1917+00$ were made by T. H. Hankins as part of another program. Details of the observations are described elsewhere (Nowakowski and Hankins 1985, Nowakowski 1991b).

\section{Results}

To achieve consistent results, the observations of the four pulsars were processed using essentially the same technique. The following paragraphs present the results of the analysis, together with a short discussion.

\section{PSR 1917+00}

Single pulses from this pulsar, when integrated over several hundred periods, form an average profile with three distinct components. It is a typical example of a profile in which both core and conal components are visible (figure 1a, solid line). The longitudinally-resolved power spectrum indicates that the brightness of the central component is modulated in a different way than that of the outer components. Amplitudes of individual pulses vary greatly from pulse to pulse, but subpulses have relatively stable phases (line with dots on figure la shows the histogram of occurrence of the maxima). Occasional bursts of strong pulses can be noticed (figure 1d). When individual pulses are integrated separately in the data segments where pulses are weak, an average profile with three components is obtained, in which the two first components are almost equal in brightness and the third component is about half of the other two. The average profile of the segments containing strong pulses is significantly different and represent the second mode. A simultaneous fit of three Gaussian components to the average profile shows that the conal components are stronger than in the previous case and shifted to the center of the profile. Although stronger than in the first mode, these components are still many times weaker than the central component. It becomes not only extremely bright but is also displaced towards later phase (figure 1c shows both profiles normalized). Switching between the 


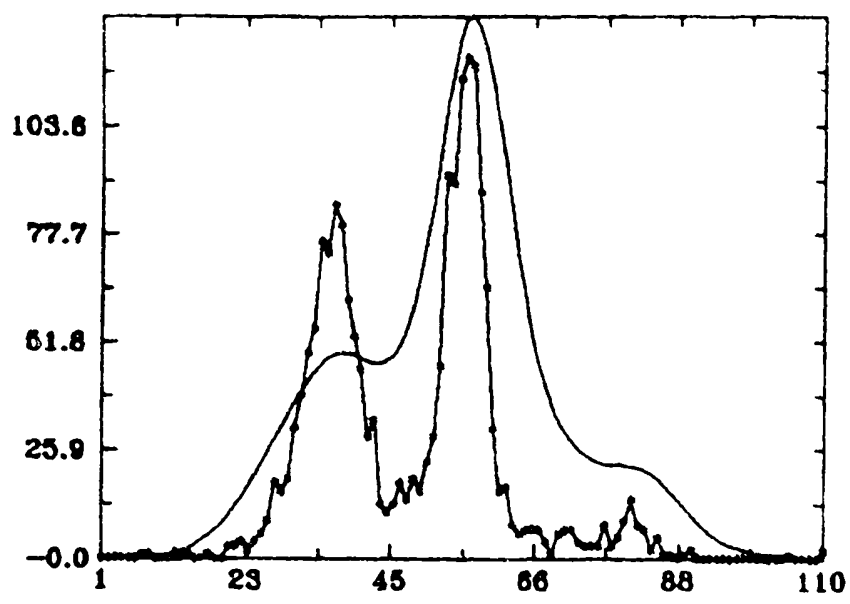

Figure 1a. The average profile of all data for PSR $1917+00$ (solid line) and a histogram showing how often individual pulses have a maximum at a given phase.

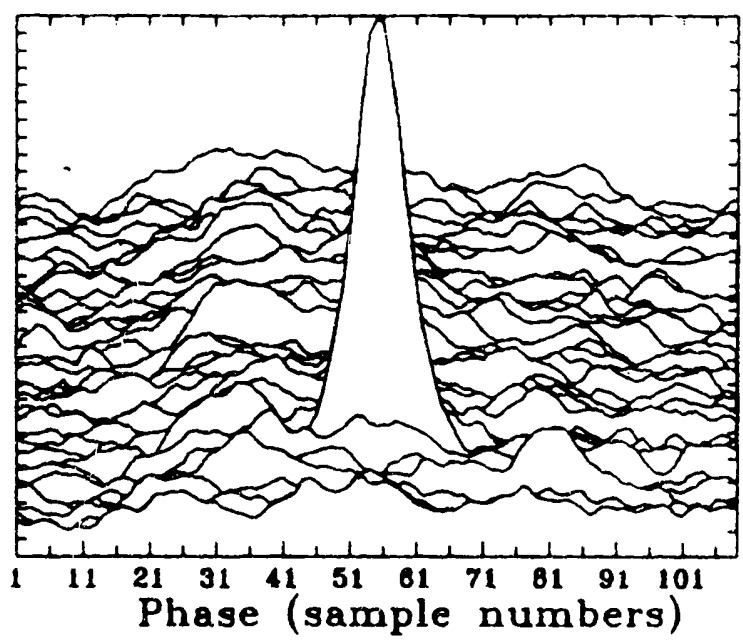

Figure 1b Several consecutive single pulses. One sțrong pulse is present, all other pulses are much weaker and belong to the normal mode.

two modes is extremely rapid and the pulsar may switch to the strong mode for only one period (figure 1b). During switching from weak to burst mode the width of the average profile decreases as a result of the displacement of the outer cumponents towards the center of the profile. At the same time the width of the central component decreases and its phase is delayed, which may be a result of retardation. The behavior of the three components, when combined with the geometry of the cone in which the radiation originates, suggests that the burst mode may be generated in deeper layers of the magnetosphere (Nowakowski and Hankins 1985).

It is very likely that this kind of mode switching found in PSR 1917+00 is not unique but is more general and can be found in other pulsars. There are, for example, similarities in this respect between this pulsar and at least two other stars: PSR 1926+18 (Ferguson et al. 1981) and PSR 0329+54 (Bartel et al. 1982). In both of them

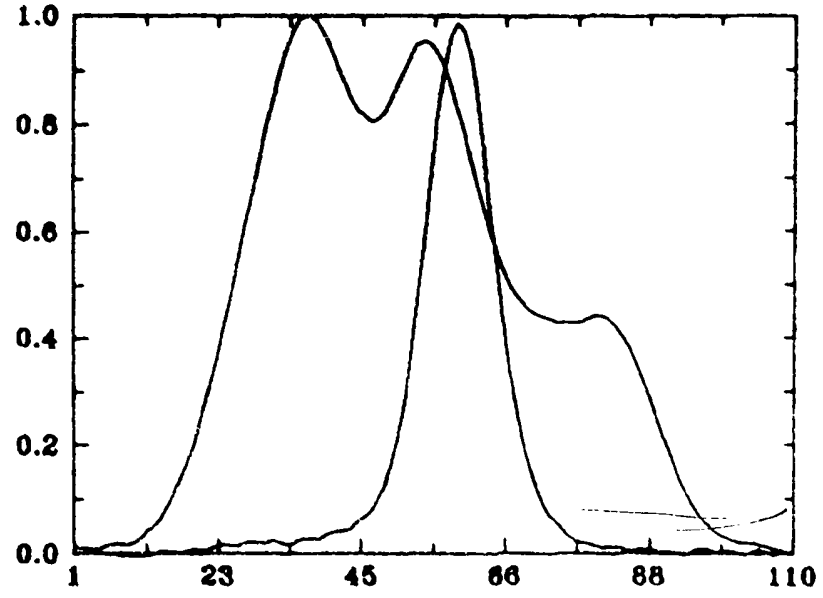

Figure 1c Average profiles of the weakest and strongest pulses, both normalized. The middle component in the strong mode arrives later than in the weak mode.

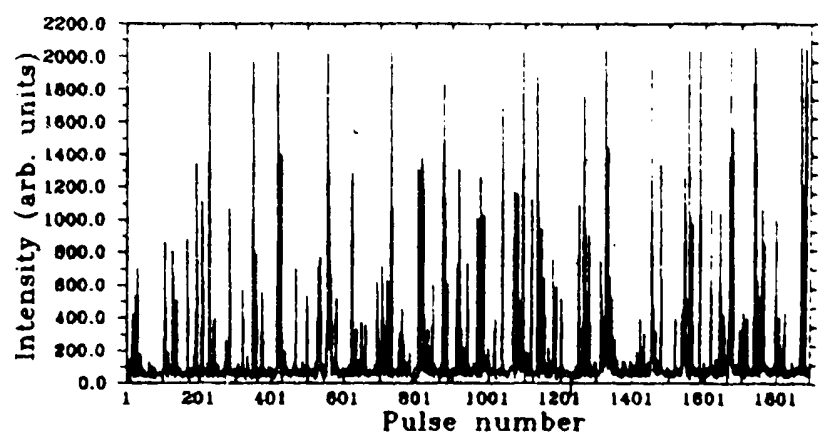

Figure 1d Intensity of individual pulses vs. pulse number. The majority of pulses are weak and short segments of strong pulses are visible.

in one mode the profile is wider than in the other because the outer components move toward the center of the profile. Recent results on the intensity dependence of the pulse profile of PSR 0329+54 (McKinnon and Hankins 1992) indicate that the core component in this pulsar arrives earlier when it is stronger. This seems to be opposite to what we observe in PSR $1917+00$ but the data used for that analysis was short and during that short observation the pulsar apparently did not switch to another mode. Therefore we still can not say how exactly the core and conal components interact during the mode switch.

There is a strong similarity in the behavior of the core component in PSR $1917+00$ and PSR $0611+22$ during mode switching: in both pulsars the core components arrive later when the pulsars are in the strong mode. In both pulsars the width of the core component decreases in the strong mode. In PSR 0611+22 however no conal components are present, and again we can not draw any conclusions about interaction of the core and conal components. 
PSR 0611+22

PSR $0611+22$ has only one component in the average profile and has been identified as core single (Rankin 1986). In that class of pulsars mode switching was not known and also that star was not suspected as a candidate for mode switching. This pulsar was known for several years as the one with the largest timing noise (Helfand et al. 1980, Gullahorn and Rankin 1982).

Single pulses from this pulsar exhibit an interesting property: their brightness changes from pulse to pulse, and in short data strings nothing special can be seen. In longer data strings however a well defined systematic property is clearly visible. Figure $2 b$ shows the unsmoothed amplitudes
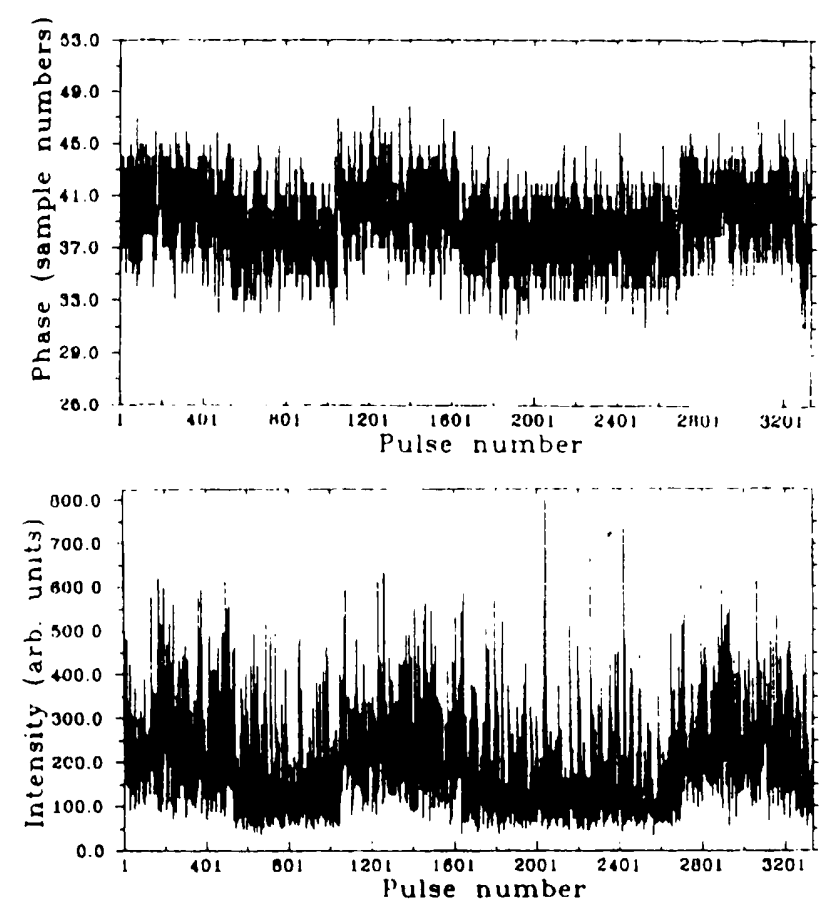

Figure 2 PSR 0611+22. Intensity of the single pulses (lower panel, a) and phases (upper panel, b) plotted against the pulse number. Correlation between the intensities and phases of the individusi pulses is visible. Integration of pulses in segments containing weak or strong pulses results in two average profiles that have different widths and phases (segments of strong pulses form a narrow profile arriving later than weak pulses that form a wider profile).

of 3332 single pulses from my data where segments of alternating stronger and weaker pulses can be seen. When this is combined with the phases of these pulses (figure 2a), it becomes apparent that there are two different states of single pulses that should give two different average profiles. Indeed, integration of the single pulses in segments containing mostly strong pulses results in an average profile that is stronger, narrower and with the peak at a later phase than the average profile of all data. On the other hand, integration in segments containing mostly weak pulses results in an average profile that is weaker, wider and with its peak intensity earlier than in the average profile of all data.

Both average profiles are single and nearly perfectly Gaussian. These two profiles represent exactly what we used to understand as mode switching in pulsars - two stable forms of the average profile. This is the first object with mode switching, which in both modes have a single, nearly perfect Gaussian profile. The two modes differ dramatically in phase and amplitude, and I have found very significant correlation between the amplitude and phase of the profiles in the two modes at $\mathbf{4 3 0}$ and $1410 \mathrm{MHz}$ : stronger individual pulses arrive at a later phase. Our observations at higher frequencies give similar results. This seems to indicate that also in this pulsar the stronger mode originates closer to the surface of the neutron star (Wolszczan, Cordes, and Nowakowski 1991).

The timing noise reported for this pulsar by other authors (Helfand et al. 1980) may be related to the mode switching effect described above. The typical time that the pulsar spends in one of the modes is slightly longer than the typical integration time used in timing observations. As a result, an average profile obtained during timing observations contained single pulses belonging to both modes. When strong pulses dominated the average profile, the phase of that profile was delayed and when weaker pulses prevailed, the average profile had its peak intensity earlier.

\section{A hidden component in PSR 1133+16}

PSR $1133+16$ has been known for many years as an example of a radio pulsar with two well separated conal components in the average profile.

Backer (1973), analyzing his $606 \mathrm{MHz}$ data noted there was a weak ordering process which correlated the emission in short series of pulses, consists of single subpulses in component I or II, subpulses in both components and pulses with no emission. Then Helfand et al. (1975) argued that the saddle region acted as a third component in a type of mode-changing phenomenon, which could have several possible states (modes). They also found that a stable form of the average profile (correlation coefficient of 0.9995 ) from this pulsar could be obtained when $\sim 2500$ consecutive individual pulses were integrated. According to its average profile and the behavior of its individual pulses Rankin (1986) has classified this source as conal double.

The power spectrum of the (integrated over the pulse window) intensity indicated modulation with period $\sim 0.027$ cycles $/ P_{1}$, corresponding to $\sim 45$ seconds (Backer 1973): The longitude-resolved modulation index for this pulsar was higher in the wings 
of the profile than in the center, slightly different for the two components and had also a maximum between the components (Taylor, Manchester, and Huguenin 1975). Sporadic drift of subpulses towards later longitudes in the trailing half of the profile was also reported (Taylor, Manchester, and Huguenin 1975).

Most of the published data for this pulsar show there is a notch in the saddle region between the two strong components that could be visible in both short and long integrations, over a wide frequency range (275 MHz: Taylor et al. 1975; 400 and $430 \mathrm{MHz}$ : Helfand et al. 1975, Taylor et al.1975; $606 \mathrm{MHz}$ : Backer 1973; $1404 \mathrm{MHz}$ : Stinebring et al. 1984).

Single pulses show, as it was reported by other authors earlier (Taylor, Manchester, and Huguenin 1975), sporadic drift of subpulses in the second part of the profile, predominantly towards the trailing edge of the profile. I have found the drift rate $\sim 0.6 \mathrm{deg} / P_{1}$ in the second component when the subpulses drift towards later phases and $\sim 0.5 \mathrm{deg}$ in the first component during a similar drift. Since this drift is sporadic (in my data I have found only a few examples where it could be seen) and very short (4-12 $\left.P_{1}\right)$, it could not be responsible for the peak in the (integrated) power spectrum reported by other authors. The longitude-resolved power spectra of my data for this pulsar are different for different parts of the average profile-both the leading and trailing edge show no features in the corresponding spectra, except for a sharp maximum at $\sim 0.0$ $c / P_{1}$. Power spectra for the saddle region and part of the second component of the profile have several features corresponding to faster modulation. The most prominent feature is located at about 0.03 $c / P_{1}$, corresponding to $\sim 32$ pulsar periods, which is equivalent to about 38 seconds. I have calculated several longitude-resolved spectra for this pulsar, for different block lengths and conclude that this difference between the spectra of different parts of the average profile is always present.

I have integrated the single pulses from this pulsar in three different ways.

First, each 100 successive pulses were integrated and this gave a set of average profiles. Despite such short integration, in most of them the morphology of the saddle region indicated the existence of the middle component. Indeed, both individual and simultaneous fitting of three Gaussian curves supported that hypothesis.

Second, individual pulses were integrated in several intensity bins. This also gave a set of average profiles that again contained a feature in the saddle region that suggested that the third component could be hidden in that part of the profile. As in the previous case, individual and simultaneous fitting of three Gaussian curves gave results supporting the idea of three components in the average profile. As figure 3 shows, it is possible that the phases of the three components depend on the intensity, at least in the low intensity profiles. This however requires further studies to be sure that the effect is real.

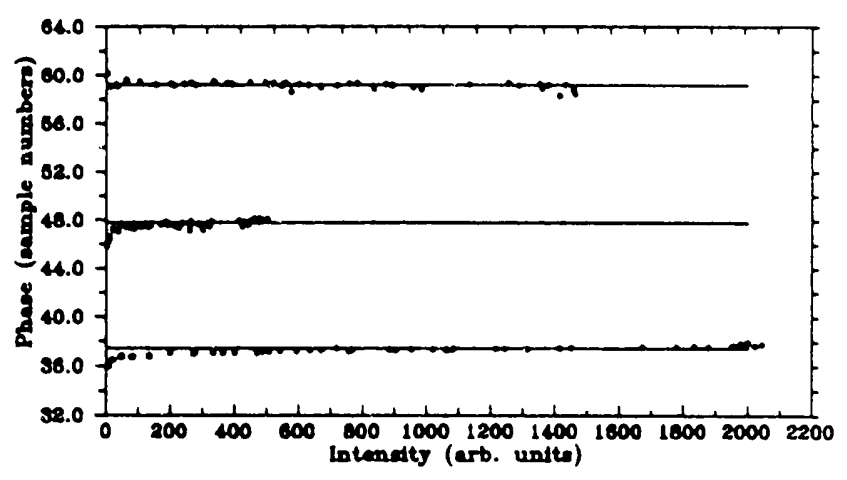

Figure 3 PSR 1133+16. Phases of the components obtained from simultaneous fits of three Gaussian curves to the average profiles (see text) plotted against the maximum intensity of the profiles used for fitting. There seems to be a tendency in the first (lower) and middle components to arrive earlier when the pulses are weak.

Third, a histogram of the longitude of maximum intensity in a sequence of individual pulses was calculated. It contained two peaks but the second one, corresponding to the second conal component was asymmetric in the saddle region. I have chosen three longitude subwindows and single pulses having their maximum intensity in these subwindows were integrated separately, giving three average profiles. One of these profiles clearly shows the presence of three components, and the middle component is strong enough to be seen separately (figure 4c).

Inspection of the single pulse data shows that one can easily identify strings of data, typically 20 $40 P_{1}$ long, where the second (middle) component is predominantly weak or strong. I have separated my data into two sets containing these two different types of pulses and integrated pulses in each of them. As a result I have obtained two average profiles that could be regarded as two modes. This problem, however, requires further study, and more data is necessary to have better statistics.

It is very interesting to note that observations of this pulsar at very high frequencies $(24 \mathrm{GHz}$, Sieber and Wielebinski 1987) have revealed that only the first (conal) component was still present, the other conal component disappeared totally, and there was a weak indication of a component 3.5 degrees later than the first component. This could not be the second conal component, since it should be at a distance of about 5.3 degrees later than the first one. This was the reason why these authors treated that feature as spurious. After appropriate rescaling to 


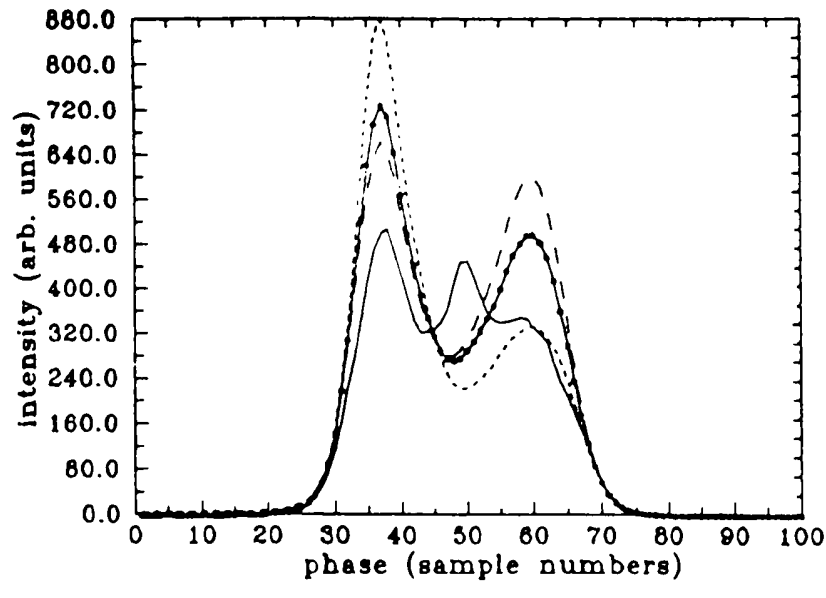

Figure 4a Individual pulses from PSR $1133+16$ were integrated in phase subwindows (see text), resulting in three different average profiles. Figure $4 \mathrm{a}$ shows them with the average profile (line with dots). Figures $4 b, 4 c$, and $4 d$ show the same average profile of all data together with each profile mentioned above. Figure $4 \mathrm{c}$ shows the profile in which the middle component is clearly visible (solid line).

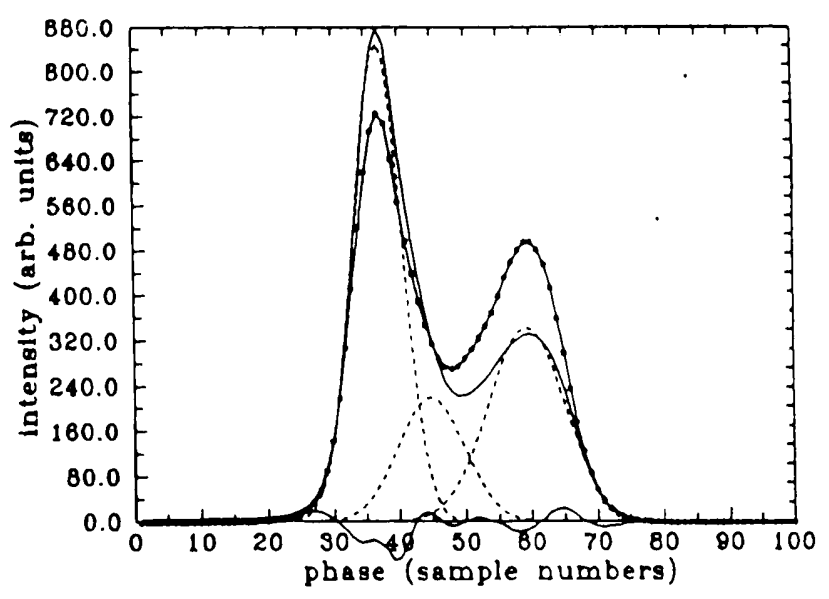

Figure $4 b$

$430 \mathrm{MHz}$, I find that the position of that feature in the 24-GHz profile corresponds to the position of the middle component that I have found in my $430-\mathrm{MHz}$ data. It is tempting to conclude then that what they could see was the third (middle) component, whose spectrum apparently should be slightly less steep than that of the third component.

\section{PSR 0540+23}

Single pulses from this pulsar, when integrated over a suitable period of time, form an average profile that looks like a typical representative of stars with only one component in the average profile. This, together with the fluctuation characteristics reported by other authors, has prompted Rankin (1986) to include this pulsar in the group of stars with core single profiles.

The most important conclusion that can be

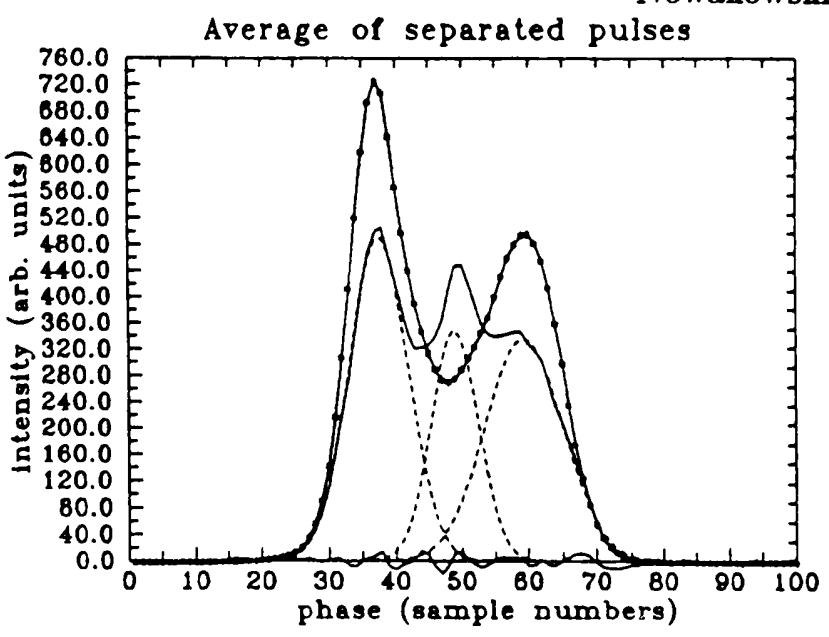

Figure 4c

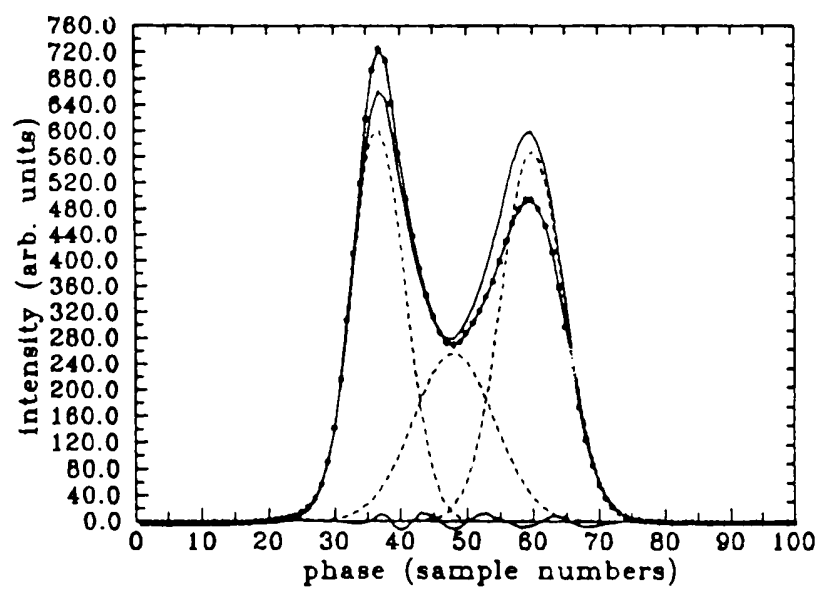

Figure 4d

drawn from the analysis of 6961 single pulses from my data on this pulsar is that this pulsar does not have a single profile! There is a second component on the trailing edge of the profile that appears sporadically, but it can be actually seen in the data and then the average profile for that segment of the data is double. Many individual pulses from this pulsar have two or three subpulses and that trailing component can be seen in short series of only a few consecutive pulses. This component is very clearly visible when only the weakest pulses are integrated.

Still another component can be seen at the beginning of the pulse window. It appears sporadically as a short train of subpulses at that phase, often together with subpulses that are present at the position of the second or third (trailing) component. Subpulses that form this component may have a stable phase during sequences of a few pulses but also drifts that end in the position of the middle component are observed.

Generally, drift of subpulses sometimes stops at certain phases that do not necessarily coincide with the positions of the components that can be identified from the average profile. Integration of the indi- 
vidual pulses having their maxima in a few selected subwindows within the pulse window results in average profiles that have two or three components. Those components are outside the subwindows selected for integration, thus can not be regarded as a product of the integration procedure.

The main component of the profile that was thought to be the core component behaves in a way that allows one to suspect that it may be in fact a conal component. This may be supported by the fact that while core components in other pulsars exhibit usually little or no ordered modulation, in this star drifting subpulses are clearly present. The drift rate is variable, from very slow to relatively fast, nevertheless organized drift of subpulses is present in most of the data. Figure 5 shows two examples when such a drift is present. Occasionally drift of subpulses can be seen across most of the pulse window and if it happens, this pattern is not repeated in subsequent pulses that exhibit a different behavior.

The behavior of the subpulses described above is repeated throughout the data and this allows us to include this pulsar to a category of those stars that exhibit mode switching. Details will be described in a subsequent paper.

\section{Discussion}

\section{Core and conal radiation}

Many observational facts indicate that the radiation from pulsars comes from a hollow cone. There are reasons to believe that the core and conal components identified by Rankin (Rankin 1986 and the references therein) represent two distinct physical radiation mechanisms.

Core radiation can be found in all kinds of profiles, beginning with the simplest, single profiles, in which only one component is visible. It may be present in triple and multiple profiles, in which it is located close to the center of the profile. About 60 percent of the known pulsar population are pulsars with core-dominated profiles. This kind of radiation then seems to be the most prototypical of pulsar emission.

Other components in the average profiles are classified as conal and have different characteristics.

Parts of the average profiles corresponding to the core components usually have flat, featureless power spectra that indicate there is little ordered modulation in the radiation that forms these components.

The core radiation also usually exhibits no nulling and no evidence of any sort of mode changing. It seems, however, that mode changing is not



Figure 5 PSR 0540+23. Lower part of the figure shows 100 consecutive pulses, with the intensities on the right side. The upper part presents the average profile (line with dots) and profiles obtained from integration in three phase subwindows. All profiles are normalized.

totally "forbidden" in the core dominated profiles. Very clear evidence for mode changing has been found in PSR 0611+22, which has a single core profile (Wolszczan, Cordes, and Nowakowski 1991). In other pulsars the core component is clearly involved in mode changing (e.g. PSR 0329+54, Bartel et al. 1982) or even seems to trigger the whole process as in PSR 1917+00 (Nowakowski and Hankins 1985).

The relatively small angular width of the core components suggests that they are emitted very close to the stellar surface.

On the other hand, the conal components often have drifting subpulses, and their angular width suggests that the location of the emitting region is relatively far away from the stellar surface. These 
components are affected during a mode changetheir phases and brightness change, resulting in a different morphology of the average profile.

There is a group of pulsars with two components in their average profile. They have conal profiles and the core component seems to be absent. This may be a result either of real absence or of weakness of the core radiation, but such a situation may arise when our line of sight merely misses (latitudinally) the central lobe of core emission. Obviously, both alternatives may be true. The saddle region between these two conal components then might contain an unseen component. In several pulsars the saddle region has fluctuation characteristics virtually identical with those of the core components in pulsars with triple and multiple profiles. Rankin (1986) concludes then that conal double profiles should be regarded as triple in spite of the fact that the third component can not be seen.

Such an unseen middle component has been isolated in the profile of PSR $1133+16$, as described above. It would be important to know if PSR $1133+16$ is an exception or typical in this class of pulsars, therefore further studies of such profiles is desirable.

\section{Mode switching and nulling}

Mode switching manifests itself as a quasi-periodic change of the pulsar signal, when individual pulses are integrated over a suitable period of time with adequate signal to noise ratio. Most of the pulsars known at the present time are thought of as possessing stable form of the integrated pulse profile and only about a dozen are known (out of more than 400 pulsars known) as those which have more than one stable profile (on different timescales, typically several hundred pulsar periods). The first pulsar with this type of behavior was found by Backer in 1970, then other pulsars were found in subsequent years (see Rankin 1986, for references). It seemed to be quite possible that this effect could be present in certain types of pulsars, in particular in those having triple and multiple profiles. Our recent results on PSR $0611+22$, however, indicate that also singlecomponent pulsars may have this effect (Wolszczan, Cordes, and Nowakowski 1991).

Mode switching is currently interpreted as reorganization of core and conal emission from pulsars and/or as radiation emitted from different layers of the magnetosphere, located at different altitudes within the emission cone (e.g. Bartel et al. 1982, Nowakowski and Hankins 1985). Some details concerning the effect are known for several pulsars (see, for example, Rankin 1986, for a review) but the mechanism itself that produces what is observed is poorly understood and requires further studies. Pulse nulling is similar to mode switching in the respect that in both phenomena all components of the average profile are affected at the same time.

Fluctuation and polarization characteristics of pulsar radiation indicate that there are two distinct radiation mechanisms responsible for core and conal radiation. On the other hand, mode switching and nulling seem to indicate that both kinds of radiation are affected at the same time during the process of mode switching or switching off in nulling. Is this then just one radiation mechanism or two that are related to each other? Our results for PSR 1917+00 presented here suggest that core and conal emission change simultaneously when mode switching takes place and thus are related.

Acknowledgment: This research was supported by a Cottrell College Science Grant from the Research Corporation. The Arecibo Observatory is operated by Cornell University under a management agreement with Cornell University. 